\title{
Evaluation of antioxidant activities of saffron stigma and spath as by-product of Crocus sativus L.
}

\begin{abstract}
The present study aimed to estimate and compare, using in vitro assays, the antioxidant activities of alcoholic extracts of saffron stigma and spath as by-products of the saffron crop. The antioxidant activity was evaluated using in vitro assays such as total antioxidant capacity, DPPH free radical, Scavenging of $\mathrm{H}_{2} \mathrm{O}_{2}$, FRAP and $\beta$-carotene bleaching assay. The results allowed us to evidence the presence of products with antioxidant and bioactive capacities in spaths and stigmas of Crocus sativus. The response of each extract depends on the type of the test. Extract from spaths protect $\beta$-carotene from bleaching more than the extract of stigmas and extract from stigmas revealed a significant reducing power activity than that of spaths. As a result, we can consider these extracts especially that of spaths like a new source of antioxidant compounds.
\end{abstract}

Keywords: antioxidant activity, spath, stigma of saffron, Crocus sativus, by-product
Volume 3 Issue 4 - 2018

\author{
Lahmass I, Ouahhoud S, Elyoubi M, Benabbas \\ R, Sabouni A, Asehraou A, Saalaoui E \\ Department of Biology, Laboratory of Biochemistry and \\ Biotechnology, Morocco
}

Correspondence: Lahmass I, Mohamed Ist University, Faculty of Sciences, Department of Biology, Laboratory of Biochemistry and Biotechnology, Morocco, Tel, +2I 2629530 036,

Email iliass.lahmass@gmail.com

Received: August 12, 2018 | Published: September 12, 2018

\section{Introduction}

Oxidative stress reflects an imbalance between the systemic manifestation of reactive oxygen species and a biological system, potentially leading to damage. ${ }^{1}$ An antioxidant is a molecule that inhibits the oxidation of other molecules. There is many types of antioxidants include enzymes (superoxide dismutase, catalase, glutathione peroxidase and reductase), glutathione and vitamins as vitamin $\mathrm{A}$, vitamin $\mathrm{C}$ and vitamin E. ${ }^{2}$ Antioxidants scavenge and control the formation of free radicals thereby preventing oxidative damage to cellular components arising as a consequence of chemical reactions involving reactive oxygen species. ${ }^{3}$

Oxygen radicals induced oxidative stress, is believed to be a principal factor in various degenerative diseases, such as atherosclerosis, ${ }^{4}$ cancer $^{5}$ and gastric ulcer. ${ }^{6}$ Numerous natural compounds occurring in plants as antioxidants, have been identified as free radicals or active oxygen scavengers. ${ }^{7}$

Saffron harvested from the dried stigmas of Crocus sativus L. (Iridaceae) flowers is used mainly as a spice for flavouring and colouring food, in alcoholic and non-alcoholic beverages, as a perfume and as a herbal medicine. Stigma of saffron was considered as red golden and the most expensive spice in the world. Since antiquity, saffron was generally used as a spice for culinary purposes and food colorant, nevertheless, in traditional medicine, applications of saffron were for treatment of various disorders as well as insomnia, liver disease, menstruation disorders, pain, digestive ailments and as stimulant, aphrodisiac and antidepressant. ${ }^{8}$ It has been demonstrated that extract from saffron and its active compounds, including crocin and safranal, have been shown a very interesting properties, to possess antioxidant, ${ }^{9}$ anticancer and anti-inflammatory, ${ }^{10}$ and memoryimproving properties. ${ }^{11}$ Saffron extracts deploy antihyperlipemic activity, ${ }^{12}$ and hyperglycemic and hypoglycemic effects. ${ }^{13,14}$ In recent times, several studies have been done to evaluate the antioxidant capacity of different reproductive parts of Crocus sativus and they showed that saffron plant and it active constituents such as crocin, carotenoids showed a significant antioxidant activity. ${ }^{15,16}$

The aim of our work was to investigate and compare, for the first time, the ethanolic extract of dried and powdered spaths as a new natural source of bioactive compounds, with that of stigmas of Crocus sativus plant using different antioxidant tests, such as, total antioxidant capacity TAC, DPPH free radical, Scavenging of $\mathrm{H}_{2} \mathrm{O}_{2}$, reducing power determination FRAP and $\beta$-carotene bleaching assay. Furthermore, the total polyphenols and total carotenoid content were also measured from extracts of plant.

\section{Materials and methods}

\section{Chemicals}

1,1-diphenyl-2-picrylhydrazyl (DPPH), hydrogen peroxide $\left(\mathrm{H}_{2} \mathrm{O}_{2}\right)$, ammonium molybdate, $\beta$-carotene and ethylenediaminetetraacetic acid (EDTA), Tween 80, potassium ferricyanide (III), Butylated hydroxytoluene (BHT), ascorbic acid, sodium phosphate, trichloroacetic acid (TCA), galic acid, $\mathrm{H}_{2} \mathrm{SO}_{4}$, sodium phosphate, Folin-Ciocalteu reagent and ferric chloride were purchased from Sigma-Aldrich (Steinheim, Germany)

All other reagents were of analytical grade.

\section{Preparation of the saffron extract}

Saffron plants have been cultivated in the region of Oujda (East of Morocco) free of any chemical treatments; the different parts of the plant were collected manually between October and November.

Dried milled powder of various piece of saffron $(1 \mathrm{~g})$, were macerated for 24 hours in $20 \mathrm{ml}$ of ethanol at room temperature. The extract was filtered using filter paper then concentrated under vacuum using the rotary evaporator. The extract was chilled in refrigerator in $4^{\circ} \mathrm{C}$ until use.

\section{Determination of total phenolic compounds}

Total phenolics of extracts from Crocus sativus were determined according to method of Lamien-Meda et al. ${ }^{17}$ with some modifications, using Folin-Ciocalteu reagent and the results were expressed as micrograms of gallic acid equivalents (GAE) per milligram of extract. A standard calibration curve was designed $(\mathrm{y}=0.1918 \mathrm{x}-0.2183$ $\left.\mathrm{r}^{2}=0.9576\right)$. 


\section{Determination of total carotenoid content}

The quantitation of total carotenoid content was performed by the method of Lee ${ }^{18}$ with some modifications. Briefly, $500 \mathrm{mg}$ of powder plant was placed with $30 \mathrm{ml}$ of hexane/acetone/ethanol $(\mathrm{v} / \mathrm{v}$; $15 / 7,5 / 7,5)$ with stirring and at room temperature. After 10min, extract was centrifuged at $4500 \mathrm{rpm}$ for $5 \mathrm{~min}$. The supernatant was recuperated the volume was and made up to $50 \mathrm{ml}$ with the extraction solvent. Absorbance was read at $450 \mathrm{~nm}$.

The total carotenoid content was calculated as following formula:

Total carotenoid Content $(\mu \mathrm{g}$ per gram $)=((\mathrm{OD}$ of sample*Volume in $\mathrm{ml}) /(\mathrm{EC} *$ Sample Weight in grams $)) * 100$

$\mathrm{EC}=2592$ (extinction coefficient of $\beta$-carotene in petroleum ether).

\section{Determination of total antioxidant capacity}

The antioxidant activity of the extracts was evaluated by the phosphomolybdate method of Prieto et al. ${ }^{19} \mathrm{An}$ aliquot of $0.3 \mathrm{ml}$ extract was added to $3 \mathrm{ml}$ of reagent solution $\left(0.6 \mathrm{M} \mathrm{H}_{2} \mathrm{SO}_{4}, 28 \mathrm{mM} \mathrm{Na}_{3} \mathrm{PO}_{4}\right.$ sodium phosphate and $4 \mathrm{mM}$ ammonium molybdate) and incubated in a water bath at $95^{\circ} \mathrm{C}$ for $60 \mathrm{~min}$. The absorbance of the solution was measured at $695 \mathrm{~nm}$ against blank after cooling to room temperature. Ascorbic acid served as standard $\left(10.975 \mathrm{x}+0.9945 \mathrm{r}^{2}=0.9986\right)$.

\section{Scavenging effect on DPPH free radical}

The scavenging effect on 2,2-diphenyl-1-picrylhydrazyl (DPPH) free radical was assessed spectrophotometrically by the method of Sánchez-Moreno et al., ${ }^{20}$ with some modifications. Briefly, $50 \mu 1$ of sample was added to $1950 \mu$ l of freshly prepared DPPH $(25 \mathrm{mg} / \mathrm{l}$ in methanol). After $30 \mathrm{~min}$ of incubation in the dark at room temperature, the absorbance was read at $517 \mathrm{~nm}$ against a blank sample consisting of $1950 \mu 1$ of DPPH solution and $50 \mu 1$ of methanol. Ascorbic acid $(0-100 \mu \mathrm{g} / \mathrm{ml})$ was used as positive control.

The DPPH radical Scavenging effect was calculated using the following equation:

\section{DPPH Scavenging effect $(\%)=\left(\left(\mathrm{A}_{0}-\mathrm{A}_{1}\right) / \mathrm{A}_{0}\right) * 100$}

Where $A_{0}$ was the absorbance of the control and $A_{1}$ was the absorbance in the presence of the sample of extracts and standards.

\section{Reducing power determination (FRAP)}

The reducing power was determined according to the method of Karagozler et al., ${ }^{21}$ with modifications. Samples $(1000 \mu \mathrm{l})$ or ascorbic acid $(0-100 \mu \mathrm{g} / \mathrm{ml})$ were mixed with $2500 \mu 1$ of $0.2 \mathrm{M}$ sodium phosphate buffer $\mathrm{pH} 6.6$, and $2500 \mu 1$ of $1 \%$ potassium ferricyanide (III), and incubated at $50{ }^{\circ} \mathrm{C}$ for $20 \mathrm{~min}$. After cooling at room temperature, $2.5 \mathrm{~mL}$ of $10 \%$ trichloroacetic acid was added and the mixture was centrifuged at $3000 \mathrm{rpm}$ for $10 \mathrm{~min}$. Then the supernatant $(2.5 \mathrm{~mL})$ was mixed with $2.5 \mathrm{~mL}$ of milli-Q water and $500 \mu 1$ of $\mathrm{FeCl}_{3}$ solution $\left(0.1 \%\right.$ in $\left.\mathrm{H}_{2} \mathrm{O}\right)$. The absorbance was measured at $700 \mathrm{~nm}$. Ascorbic acid was used as positive control and the reducing power activity was expressed asmg of ascorbic acid equivalent per gram (mg $\mathrm{AAE} / \mathrm{g}$ ) of extract.

\section{Scavenging of hydrogen peroxide}

The ability of saffron extracts to scavenge hydrogen peroxide was determined according to the method of Ruch et al. ${ }^{22}$ A solution of hydrogen peroxide $(2 \mathrm{mmol} / \mathrm{l})$ was prepared in phosphate buffer $(\mathrm{pH}$ 7.4). Extract was added to hydrogen peroxide solution $(0.6 \mathrm{ml})$. The concentration of Hydrogen peroxide was determined after $10 \mathrm{~min}$ of incubation at $230 \mathrm{~nm}$. For background subtraction a separate blank sample was used. The scavenging activity of $\mathrm{H}_{2} \mathrm{O}_{2}$ was expressed asmg of ascorbic acid equivalent per gram ( $\left.\mathrm{mg} \mathrm{AAE}^{2} / \mathrm{g}\right)$ of extract.

\section{$\beta$-carotene bleaching assay}

The antioxidant activity of saffron extracts was evaluated by the $\beta$-carotene linoleate model system Tepe et al. ${ }^{23}$ In a flask, $2 \mathrm{mg}$ of $\beta$-carotene was putted and dissolved in $1 \mathrm{ml}$ of chloroform. After evaporating the chloroform, $20 \mathrm{mg}$ of linoleic acid, $200 \mathrm{mg}$ of Tween 80 emulsifier and $50 \mathrm{ml}$ of oxygenated water were added to the flask with vigorous shaking. An aliquot $(250 \mu 1)$ of this emulsion was transferred into microplate of 96 wells containing $50 \mu \mathrm{l}$ of different concentrations of the plant extracts and absorbance was determined at $490 \mathrm{~nm}$ immediately against the blank solution. BHT was used as standard. Other readings are measured at different time intervals (2, $4,6,12$, and $48 \mathrm{~h}$ ). The antioxidant activity relative after 48 hours is calculated using the following equation:

$$
\operatorname{AAR}(\%)=(\mathrm{OD} \text { of sample } / \mathrm{OD} \text { of BHT }) \times 100 .
$$

\section{Statistical analysis}

All experiments were repeated three times. Means and standard deviations (SD) were calculated using Microsoft Office Excel.

\section{Results and discussion}

\section{Total phenolic and carotenoid content}

The amount of total phenolics and carotenoid content in the two extracts of saffron plant were determined using the Folin-Ciocalteu method and method of Lee ${ }^{18}$ then the results were expressed as gallic acid equivalents and carotene equivalent respectively. Because the role occupied by polyphenols and carotenoids in protecting against free radicals and oxidative stress, and contributed directly to the antioxidant activity of plant so it's important to quantify these essential compounds. The results Table 1 showed that stigma contained a significantly higher amount of carotenoids compounds and lower content of polyphenol than spath (for polyphenols: $16.63 \pm 0.1$ and $21.36 \pm 0.025 \mu \mathrm{g} \mathrm{GAE} / \mathrm{mg}$ extract, for carotenoids: $16.132 \pm 0.015$ and $1.181 \pm 0.006 \mu \mathrm{g} \mathrm{GAE} / \mathrm{mg}$ extract respectively).

\section{Total antioxidant capacity}

The phosphomolybdenum method was founded on the conversion of molybdate ions $\left(\mathrm{MoO}_{4}^{2-}\right)$ to molybdenum $\left(\mathrm{MoO}^{2+}\right)$ by compounds with an antioxidant activity and like result a formation of a green phosphate $\mathrm{Mo}(\mathrm{V})$ composite with a maximal absorption at $695 \mathrm{~nm}$. Our data (Table 1) are expressed as the number of equivalents of ascorbic acid and showed that the capacity antioxidant total of the extract is rising with the increasing concentration of the plant extract.

\section{DPPH scavenging activity}

Antioxidant properties, especially radical scavenging activities, are very important due to the deleterious role of free radicals in foods and in biological systems. Excessive formation of free radicals accelerates the oxidation of lipids in foods and decreases food quality and consumer acceptance. ${ }^{24}$

The method is based on the reduction of the stable free radical DPPH in the presence of a hydrogen-donating antioxidant, and the formation of the non-radical form DPPH-H as result of the reaction. Data corresponding to incubations of DPPH with different concentrations of extracts of saffron and with Ascorbic acid are shown in Figure 1. 
Table I Yield, total phenolic compounds, carotenoid content and total antioxidant capacity of ethanolic extract of stigma and spath of Crocus sativus

\begin{tabular}{lllll}
\hline Sample & Yield (\%) & $\begin{array}{l}\text { total phenolic } \\
\text { compounds } \boldsymbol{\mu g} \text { GAE/mg } \\
\text { extract }\end{array}$ & $\begin{array}{l}\text { carotenoid content } \boldsymbol{\mu g} \\
\text { carotene E/mg extract }\end{array}$ & $\begin{array}{l}\text { total antioxidant capacity } \boldsymbol{\mu g} \text { AAE/ } \\
\text { mg extract }\end{array}$ \\
\hline spath & 5,8 & $21,36 \pm 0,025$ & $1,181 \pm 0,006$ & $0,069 \pm 0,126$ \\
stigma & 28,76 & $16,63 \pm 0,100$ & $16,132 \pm 0,015$ & $0,500 \pm 0,050$ \\
\hline
\end{tabular}

In our study, DPPH radical is used for evaluation of free radicalscavenging properties of different parts of saffron. The results of investigation are shown in Figure 1. Free radical scavenging activity of spath extracts was similar to that of stigma extract.

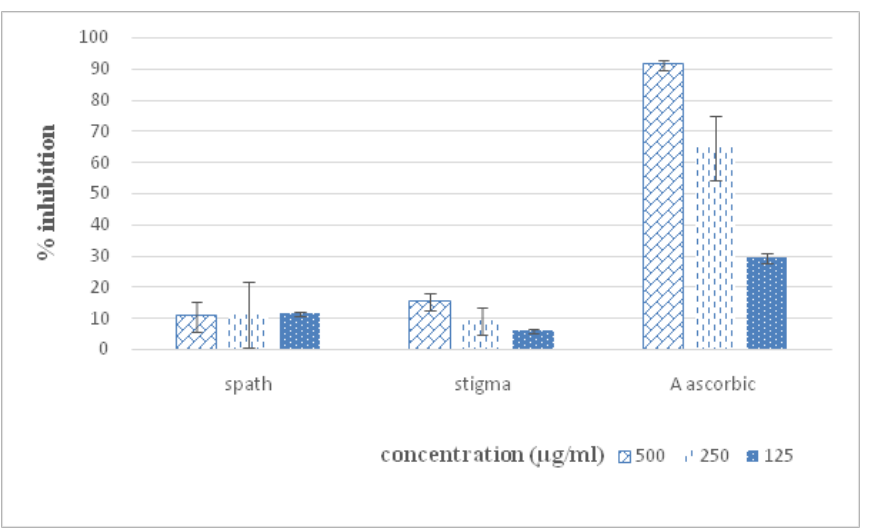

Figure I DPPH radical-scavenging activities of ethanolic extracts of Crocus stigma and spath. Ascorbic acid was used as a positive control. Results are expressed as the mean $\pm \mathrm{SD}$ of triplicate measurements.

\section{Determination of reducing activity FRAP}

The reducing power determination is based on the reduction of $\mathrm{Fe}^{3+}$ to $\mathrm{Fe}^{2+}$ by donating electron from sample or antioxidant. Increased absorbance indicates increased ability of the extracts to act as antioxidants by donating electrons.

Our data in Figure 2 showed that reducing power activity of spath was inferior to activity of stigmas 0.157 and 0.707 successively at $250 \mu \mathrm{g} / \mathrm{ml}$. The positive control showed a higher reducing activity with 0.77 at $100 \mu \mathrm{g} / \mathrm{ml}$. For that reason, our saffron extracts can stopped radical chain reaction by donating electron to free radicals and reacting with them, thus, convert them to more stable products.

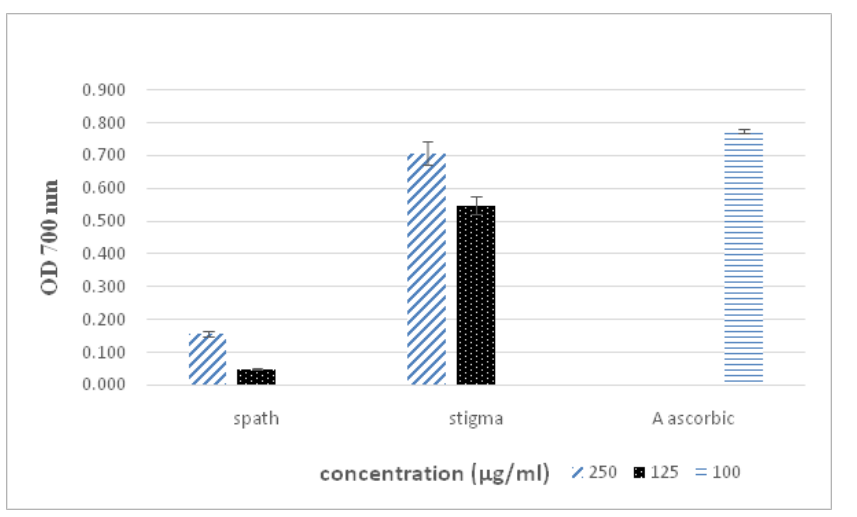

Figure 2 Determination of ferrous reducing capacity of ethanolic extracts of Crocus stigma and spath. Ascorbic acid was used as a positive control. Results are expressed as the mean $\pm S D$ of triplicate measurements.

\section{Scavenging of hydrogen peroxide}

Scavenging of hydrogen peroxide was determined according to the method of Ruch et al. ${ }^{22}$ The ability of ethanolic extract of stigma and spath to scavenging hydrogen peroxide is shown in Figure 3 and compared to ascorbic acid as positive control.

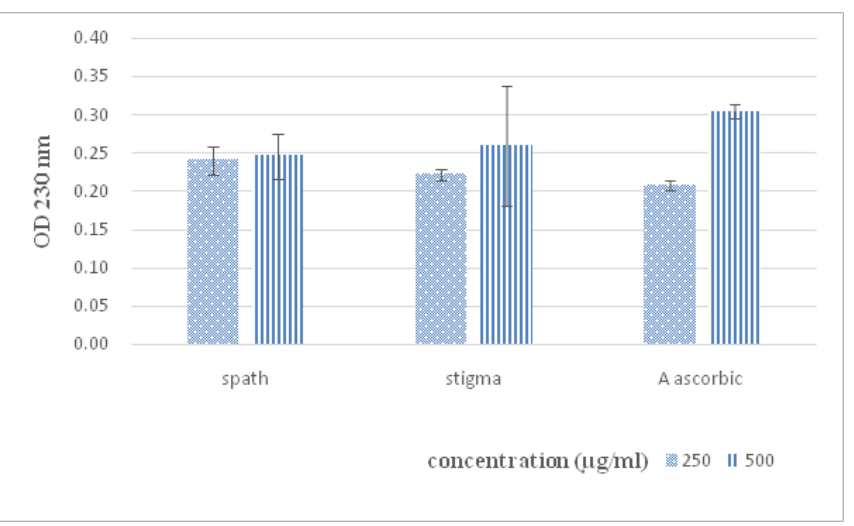

Figure 3 Scavenging activity of ethanol extracts of spath and stigma on hydrogen peroxide. Ascorbic acid was used as a positive control. Results are expressed as the mean $\pm S D$ of triplicate measurements.

The results revealed that both extracts have appreciable scavenging character in accordance with the standards and the inhibition percentage was proportional to the concentrations of each extract, with values of $379.33 \pm 0.078 \mathrm{mg}$ of ascorbic acid equivalent per gram of stigma extract and $352.00 \pm 0.029 \mathrm{mg} \mathrm{AAE} / \mathrm{g}$ of spath extract. Hydrogen peroxide is toxic to cell because it may give increase to hydroxyl radical and free radicals in the cell. ${ }^{25}$ Consequently, removing of $\mathrm{H}_{2} \mathrm{O}_{2}$ is very essential for antioxidant defense in cell or food systems. Present study revealed that the Crocus sativus ethanolic extracts are capable of scavenging hydrogen peroxide and it has been demonstrated that polyphenols, flavonoids and carotenoids compounds protect living cells against the cytotoxicity induced by hydrogen peroxide, by their orthodihydroxy phenolic structure quercetin, gallic acid ester, catechin, caffeic acid ester, ${ }^{26}$ then our extracts contain these phenolic compounds which can interact in the elimination of harmful $\mathrm{H}_{2} \mathrm{O}_{2}$ as is demonstrated recently by Baba et al., ${ }^{9}$ who reported that ethanolic extract from saffron attenuated oxidative stress in plants, yeast, and bacteria.

\section{$\beta$-carotene bleaching assay}

Figure 4 shows the ability of the ethanolic extracts from stigmas and spath of Crocus sativus to inhibit oxidation of $\beta$-carotene as measured by $\beta$-carotene bleaching method. Both extracts stopped the oxidation of $\beta$-carotene with different antioxidant activity relative: the extract of spath $(57.597 \% \pm 0.003)$ was effective and superior to that of stigma (31.604\% \pm 0.09 ). The highest activity was observed for the positive control with a $100 \% \pm 0.042$ inhibition rate.

The $\beta$-carotene bleaching system is based on the rapid discoloration 
of $\beta$-carotene, their oxidation then following by the loss of orange color in the absence of an antioxidant in the reaction medium, the system loses its orange color. The presence of extracts with antioxidant activity can prevent $\beta$-carotene oxidation by neutralization of the free radicals formed in the system by linoleic acid. ${ }^{27}$ Our study showed that the ethanolic extract of spath demonstrated higher ability to prevent the bleaching of $\beta$-carotene than that of ethanolic extract of stigma. This antioxidant activity may be due to active constituents of saffron like crocin and safranal and also by phenolic compounds. ${ }^{15}$

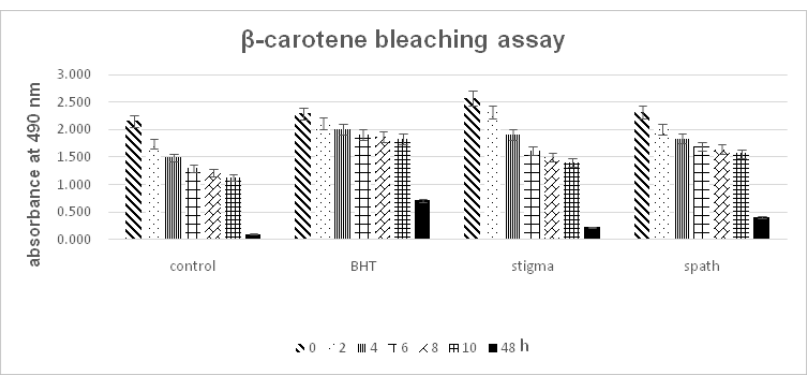

Figure 4 Antioxidant activity of saffron and spath extracts and BHT in $\beta$-carotene-linoleate system after 48 hours of incubation in the dark.

\section{Conclusion}

In summary, the data presented here show that stigma and spath extracts contain compounds possessing high antioxidant capacity. Present study showed that spaths and stigmas besides being colorant could play a role as antioxidant source; further studies are needed to elucidate the different antioxidant mechanisms. Further investigations are currently underway to investigate the isolation, quantification and identification of molecular profile.

\section{Acknowledgements}

This research is financially sponsored by the ARES "Coopération au développement". The authors are very thankful the ARES for financial assistance.

\section{Conflict of interest}

The author confirms that this article content has no conflicts of interest.

\section{References}

1. Halliwell B. Biochemistry of oxidative stress. Biochem Soc Trans. 2007;35(Pt 5):1147-1150.

2. Devasagayam T, Tilak J, Boloor K, et al. Free radicals and antioxidants in human health: current status and future prospects. $J$ Assoc Physicians India. 2004;52(10):794-804.

3. Pandey A, Mishra A, Mishra A. Antifungal and antioxidative potential of oil and extracts derived from leaves of Indian spice plant Cinnamomum tamala. Cell Mol Biol (Noisy-le-grand). 2012;58(1):142-147.

4. Steinberg D, Parthasarathy S, Carew TE, et al. Beyond Cholesterol. $N$ Engl J Med. 1989;320(14):915-924.

5. Muramatsu H, Kogawa K, Tanaka M, et al. Superoxide dismutase in SAS human tongue carcinoma cell line is a factor defining invasiveness and cell motility. Cancer Res. 1995;55(24):6210-6214.

6. Das D, Bandyopadhyay D, Bhattacharjee M, et al. Hydroxyl radical is the major causative factor in stress-induced gastric ulceration. Free Radical Biology and Medicine. 1997;23(1):8-18.
7. Zheng W, Wang SY. Antioxidant activity and phenolic compounds in selected herbs. J Agric Food Chem. 2001;49(11):5165-5170.

8. Schmidt M, Betti G, Hensel A. Saffron in phytotherapy: pharmacology and clinical uses. Wiener klinische Wochenschrift. 2007; 157(13-14):315-319.

9. Baba SA, Malik AH, Wani ZA, et al. Phytochemical analysis and antioxidant activity of different tissue types of Crocus sativus and oxidative stress alleviating potential of saffron extract in plants, bacteria, and yeast. South African Journal of Botany. 2015;99:80-87.

10. Samarghandian S, Boskabady $\mathrm{MH}$, Davoodi S. Use of in vitro assays to assess the potential antiproliferative and cytotoxic effects of saffron (Crocus sativus L.) in human lung cancer cell line. Pharmacogn Mag. 2010;6(24):309-314.

11. Abdullaev FI, Espinosa-aguirre JJ. Biomedical properties of saffron and its potential use in cancer therapy and chemoprevention trials. Cancer Detection and Prevention. 2004;28(6):426-432.

12. Asdaq SM, Inamdar MN. Potential of Crocus sativus (saffron) and its constituent, crocin, as hypolipidemic and antioxidant in rats. Applied Biochemistry and Biotechnology. 2010;162(2):358-372.

13. Elgazar AF, Rezq AA, Bukhari HM. Anti-hyperglycemic effect of saffron extract in alloxan-induced diabetic rats. European Journal of Biological Research. 2013;5(1):14-22.

14. Hemmati M, Asghari S, Zohoori E, et al. Hypoglycemic effects of three Iranian edible plants; jujube, barberry and saffron: Correlation with serum adiponectin level. Pakistan Journal of Pharmaceutical Sciences. 2015;28(6):2095-2099.

15. Assimopoulou AN, Sinakos Z, Papageorgiou VP. Radical scavenging activity of Crocus sativus L. extract and its bioactive constituents. Phytotherapy Research. 2005;19(11):997-1000.

16. Chen Y, Zhang H, Tian X, et al. Antioxidant potential of crocins and ethanol extracts of Gardenia jasminoides ELLIS and Crocus sativus L. A relationship investigation between antioxidant activity and crocin contents. Food Chemistry. 2008;109(3):484-492.

17. Lamien-meda A, Lamien CE, Compaore MM, et al. Polyphenol content and antioxidant activity of fourteen wild edible fruits from Burkina Faso. Molecules. 2008;13(3):581-594.

18. Lee HS. Characterization of carotenoids in juice of red navel orange (Cara Cara). Journal of Agricultural and Food Chemistry. 2001;49(5):2563-2578.

19. Prieto P, Pineda M, Aguilar M. Spectrophotometric quantitation of antioxidant capacity through the formation of a phosphomolybdenum complex: specific application to the determination of vitamin E. Analytical Biochemistry. 1999;269(2):337-341.

20. Sánchez $\square$ moreno C, Larrauri JA, Saura $\square$ calixto F. A procedure to measure the antiradical efficiency of polyphenols. Journal of the Science of Food and Agriculture. 1998;76(2):270-276.

21. Karagozler AA, Erdag B, Emek YC, et al. Antioxidant activity and proline content of leaf extracts from Dorystoechas hastata. Food Chemistry. 2008;111(2):400-407.

22. Ruch RJ, Cheng SJ, Klaunig JE. Prevention of cytotoxicity and inhibition of intercellular communication by antioxidant catechins isolated from Chinese green tea. Carcinogenesis. 1989;10(6):1003-1008.

23. Tepe B, Sokmen M, Akpulat HA, et al. Screening of the antioxidant potentials of six Salvia species from Turkey. Food Chemistry. 2006;95(2):200-204.

24. Min DB, Boff JM. Lipid oxidation of edible oil. Food Science and Technology. New York: Marcel Dekker; 2002:335-364. 
25. Halliwell B. Reactive oxygen species in living systems: Source, biochemistry, and role in human disease. The American Journal of Medicine. 1991;91(3C):S14-S22.

26. Nakayama T. Suppression of hydroperoxide-induced cytotoxicity by polyphenols. Cancer Res. 1994;54(7 Suppl):1991s-1993s.
27. Jayaprakasha G, Singh R, Sakariah K. Antioxidant activity of grape seed (Vitis vinifera) extracts on peroxidation models in vitro. Food Chemistry. 2001;73(3):285-290. 